\title{
Relationship between decreased expression of squamous cell carcinoma antigen 2 and E-cadherin in primary cervical cancer lesions and lymph node metastasis
}

\author{
AKIHIRO MURAKAMI ${ }^{1}$, TATSUSHI NAKAGAWA ${ }^{1}$, CHIKAKO FUKUSHIMA $^{1}$, MAYUMI TORII ${ }^{1}$, \\ KOTARO SUEOKA $^{1}$, SHUGO NAWATA ${ }^{1}$, OSAMU TAKEDA ${ }^{1}$, HITOSHI ISHIKAWA ${ }^{2}$ and NORIHIRO SUGINO ${ }^{1}$ \\ ${ }^{1}$ Department of Obstetrics and Gynecology, Yamaguchi University Graduate School of Medicine, \\ 1-1-1 Minamikogushi, Ube 755-8505; ${ }^{2}$ Department of Public Health, Yamagata University \\ Graduate School of Medicine, 2-2-2 Iidanishi, Yamagata 990-9585, Japan
}

Received May 28, 2007; Accepted September 4, 2007

\begin{abstract}
Squamous cell carcinoma antigen (SCCA) has been used for the management of squamous cell carcinoma, especially in order to evaluate therapeutic effects and monitor recurrence. Recent studies have shown that SCCA performs several biological functions and can influence the behavior of cancer cells. It is well known that altered expression of E-cadherin is involved in the process of cancer invasion and metastasis. The present study was therefore undertaken to investigate the relationship between the expression of SCCA, E-cadherin and lymph node metastasis in advanced cervical squamous cell carcinoma patients. We studied 70 patients who had undergone radical hysterectomy and pelvic lymphadenectomy for stage IB, IIA and IIB of the disease, without pretreatments. Immunohistochemistry, using monoclonal antibodies against SCCA2 and E-cadherin, was performed to examine the relationship between SCCA2 and E-cadherin expression patterns in primary cancer lesions and lymph node metastasis. There was a significant positive relationship between the two expression patterns in primary cancer lesions $(\mathrm{p}<0.01)$. Both exhibited a heterogeneous expression pattern in the primary tumor which indicated a significant relationship with lymph node metastasis $(\mathrm{p}<0.01)$. Our data clearly show that SCCA2 expression is significantly related to E-cadherin expression and that the heterogeneous pattern of SCCA and E-cadherin in primary lesions is strongly associated with the high incidence of lymph node metastasis in cervical squamous cell carcinoma. These findings suggest that SCCA2 may be involved in cancer behavior such as metastasis, and as such can be a useful marker in predicting lymph node metastasis.
\end{abstract}

Correspondence to: Dr Norihiro Sugino, Department of Obstetrics and Gynecology, Yamagata University Graduate School of Medicine, 1-1-1 Minamikogushi, Ube 755-8505, Japan

E-mail: sugino@yamaguchi-u.ac.jp

Key words: squamous cell carcinoma antigen, E-cadherin, lymph node metastasis

\section{Introduction}

Squamous cell carcinoma antigen (SCCA) was first discovered as a tumor-related protein in squamous cell carcinoma of the uterine cervix (1). SCCA has been used for the management of squamous cell carcinoma, especially for the evaluation of therapeutic effects and for monitoring disease recurrence (2). Recent molecular studies have shown that SCCA is encoded by two high homogeneous genes, SCCA1 and SCCA2, which are tandemly arrayed at the 18q21.3 locus (3). Both SCCA1 and SCCA2 belong to the serine proteinase inhibitor (serpin) superfamily $(3,4)$. SCCA1 inhibits both serine proteinases (e.g. chymotripsin) and cysteine proteinases (e.g. papain and cathepsin L, K and S), while SCCA2 inhibits only serine proteinases (e.g. chymase and cathepsin G) (5-8). Our previous studies have shown that SCCA2 suppresses apoptosis in human cancer cells, suggesting that it serves as a survival factor (9). It has also been reported that SCCA2 expression is increased in squamous cell carcinoma tissues and that SCCA2 transfectant induces rapid tumor growth in vivo $(10,11)$. These facts led us to speculate that SCCA2 may perform important biological functions, and may be involved in the regulation of cancer behaviors in squamous cell carcinoma.

A number of studies have shown that altered expression of cell adhesion molecules is involved in cancer behavior, especially in the process of invasion and of metastasis, which comprises multiple steps. The loss of expression of E-cadherin, a main cell adhesion molecule of the epithelium that mediates cell-to-cell contact, causes cancer cells in the primary tumor to detach (12-15), which is the first stage of cancer metastasis. In fact, some reports have shown that E-cadherin is related to lymph node metastasis in squamous cell carcinoma of the esophagus $(14,15)$, head and neck $(13,16)$ and skin $(17)$. However, for the uterine cervix little information is available regarding the relationship between E-cadherin expression and lymph node metastasis in vivo. Interestingly, we have recently found that the suppression of SCCA2 expression promotes cancer cell invasion and migration by decreasing E-cadherin expression in squamous cell carcinoma cells in vitro (18). It is therefore plausible that SCCA may be involved in metastasis through the alteration of E-cadherin expression. In order to 

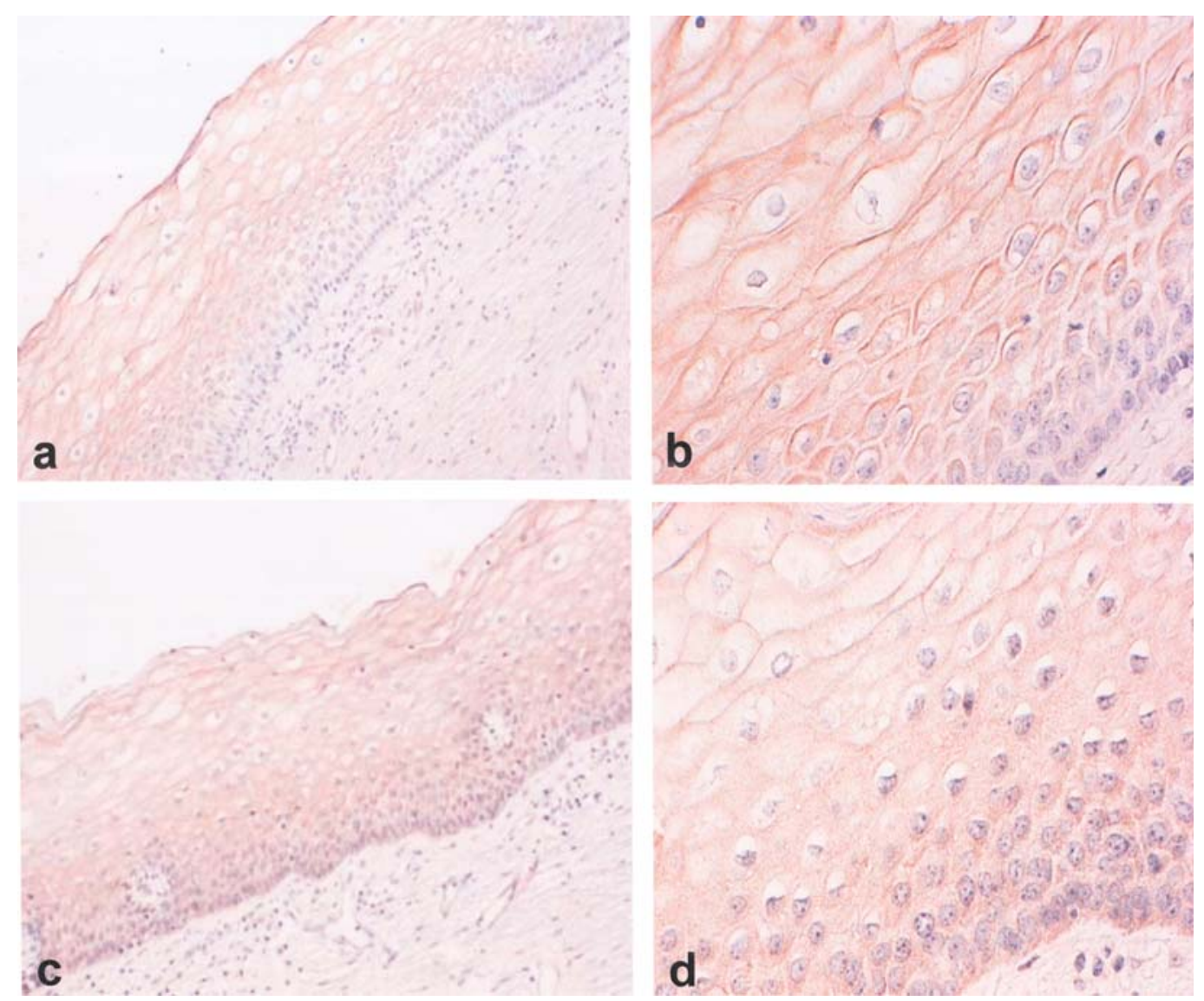

Figure 1. Immunohistochemistry for SCCA2 and E-cadherin expression in normal cervical squamous epithelium. (a) SCCA2 expressed in all epithelial layers except the basal layer. (b) Strong expression of SCCA2 observed in the cytoplasm of epithelial cells. (c) E-cadherin expressed in all epithelial layers. (d) E-cadherin expression in normal epithelial cells localized in the cytosol as well as on cell-cell boundaries. Original magnification (a and c), x100; (b and d), $\mathrm{x} 400$.

study the possibility that SCCA2 and E-cadherin are involved in lymph node metastasis, we examined the relationship between the expression of SCCA2 and E-cadherin in primary cervical cancer lesions and lymph node metastasis.

\section{Materials and methods}

Patients and specimens. Included in the study were 70 patients with squamous cell carcinoma of the uterine cervix who underwent surgery at the Department of Obstetrics and Gynecology at Yamaguchi University Hospital and its two related hospitals. Metastatic lymph nodes were also obtained from 20 patients at the time of surgery. None of the patients had received anticancer chemotherapy or irradiation therapy before the operation. All tumor tissues were obtained from surgically resected specimens. Normal squamous epithelial tissues were obtained from non-cancerous mucosa adjacent to the primary tumor in all patients.

Immunohistological staining procedures. The streptavidinbiotin-peroxidase complex technique was used for immunohistochemical staining as follows: specimens were fixed in $10 \%$ buffered formalin and embedded in paraffin. All paraffin blocks were cut into $3 \mu \mathrm{m}$-thick sections for immunohistochemistry. Series of tissue sections were deparaffinized in xylenes and rehydrated in ethanol. Samples were washed with cold phosphate-buffered saline (PBS). Endogeneous peroxidase was blocked by incubating the sections with $0.5 \%$ hydrogen peroxide in methanol for $30 \mathrm{~min}$ at room temperature. The sections were then washed three times in cold PBS. After incubation with $10 \%$ normal rabbit serum to block non-specific binding, the specimens were sequentially incubated with primary monoclonal antibody against SCCA2 (kindly provided by Dr Gary A. Silverman) and E-cadherin (Santa Cruz Biotechnology Inc., Santa Cruz, CA) at $4^{\circ} \mathrm{C}$ overnight. After incubation, they were rinsed in cold PBS and incubated for $30 \mathrm{~min}$ at room temperature with biotinylated anti-mouse $\operatorname{IgG}+\operatorname{Ig} \mathrm{A}+\operatorname{IgM}$ using the Histofine SAB PO kit (Nichirei, Tokyo, Japan) followed by streptavidin-biotin-peroxidase complex. The reaction was revealed by adding diaminobenzindinetetrahydrocholoride chromogen mixture (Sigma, St. Louis, MO). After hematoxylin couterstaining, slides were permanently mounted and analyzed for the presence and distribution of immunostaining. Results were assessed in a blinded fashion by two independent observers.

Evaluation of SCCA2 and E-cadherin expression. Evaluation was performed according to the Shiozaki et al method, with some modifications (14). Briefly, the intensity of SCCA2/ E-cadherin staining in cancer cells from cancerous tissue was compared to that in the uterine normal squamous epithelium. When SCCA2/E-cadherin staining in the cancer cells was as strong as it was in the normal epithelial cells, the intensity of the cells was defined as (+). When SCCA2/E-cadherin 

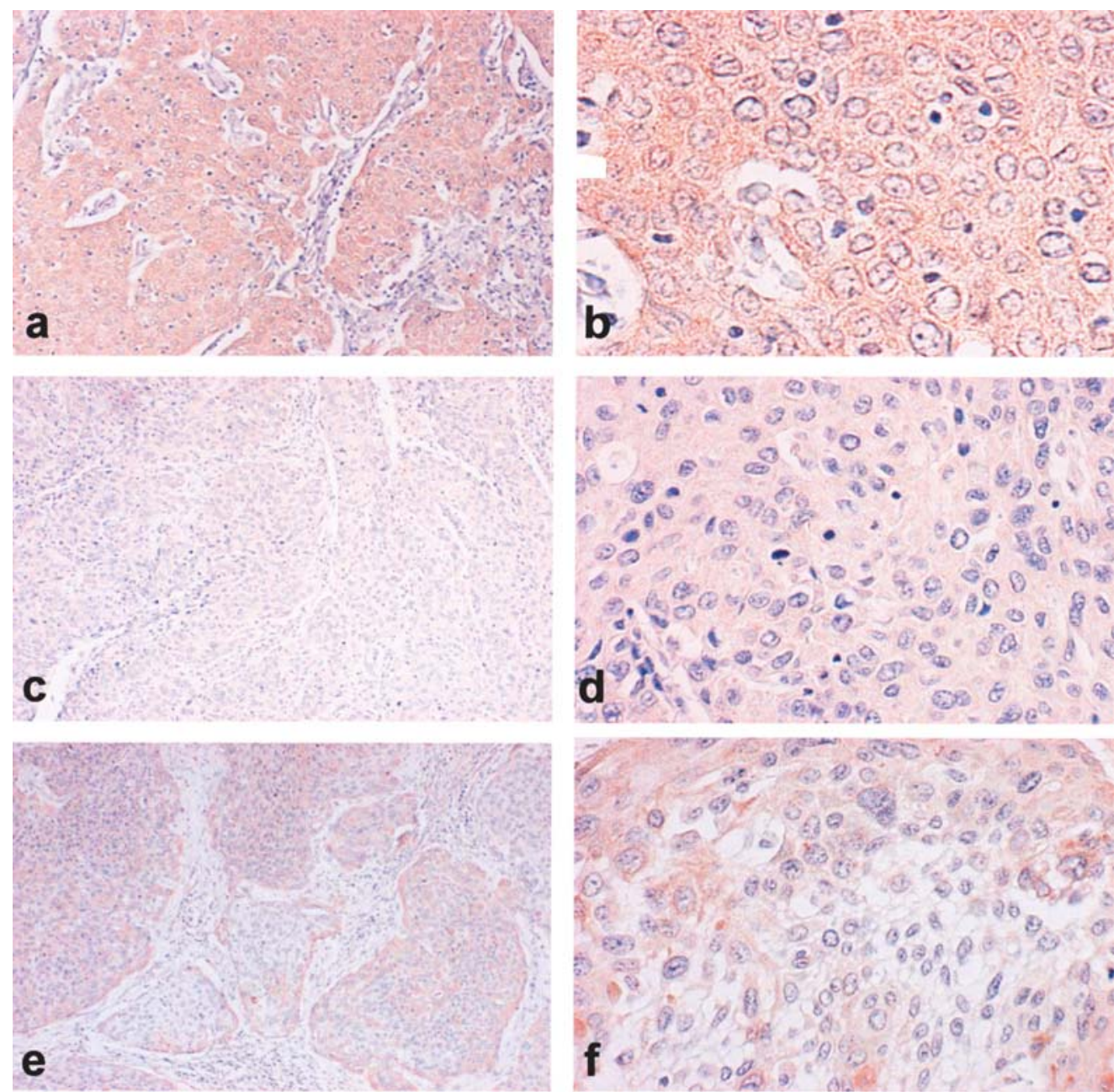

Figure 2. Expression patterns of SCCA2 in primary cervical squamous cell carcinoma by immunohistochemistry. (a, b) Strong pattern: the intensity of SCCA2 staining in cancer cells is similar to that in the normal squamous epithelial cells seen in Fig. 1. All cancer cells evaluated as (+). (c, d) W\&H pattern: SCCA2 staining in cancer cells is weaker than that in the normal epithelial cells. All cancer cells evaluated as ( \pm ). (e, f) Heterogeneous pattern: the intensity of SCCA2 staining differs from cell to cell. Some cancer cells evaluated as $(+),( \pm)$, or (-). Original magnification (a, c and e), x100; (b, d and f), x400.

staining in the cancer cells was recognizable but weaker than that in the normal cells, the intensity was defined as $( \pm)$. Loss of SCCA2/E-cadherin expression in the cancer cells was defined as (-). All cases were further classified according to the pattern of staining on representative sections of each tumor as follows: a) Strong pattern, all cancer cells in the cancerous tissues were evaluated as $(+)$; b) Weak and homogeneous $(\mathrm{W} \& \mathrm{H})$ pattern, all cancer cells were $( \pm)$, uniformly stained but more weakly expressed than in the normal squamous epithelium; c) Heterogeneous pattern, the intensity of SCCA2/E-cadherin staining differed from cell to cell and negative cells without immunostaining were included. Some cancer cells were therefore evaluated as $(+),( \pm)$ and $(-)$.

Statistical analysis. The significance of the differences in proportion was estimated for the group as a whole using the $\chi^{2}$ test, and between the two groups using Fisher's exact test with Bonferroni correction. The Spearman rank correlation test was used to assess the correlation between SCCA2 and E-cadherin expression, and their expression in relation to lymph node metastasis. The level of significance was $p<0.01$. All statistical analyses were carried out using the StatView 5.0J for Windows (SAS Institute Inc.) software package.

\section{Results}

SCCA2 and E-cadherin expression in the normal squamous epithelium. Strong SCCA2 expression was observed in the suprabasal layer of the squamous epithelium, and weak immunoreactivity in the basal layer (Fig. 1a). SCCA2 was localized in the cytoplasm of normal squamous epithelial cells (Fig. 1b). E-cadherin immunostaining was characteristically distributed at the basal and parabasal cell layer levels of the squamous epithelium. Keratinocytes in the superficial layers also showed intense immunoreactivity (Fig. 1c). E-cadherin expression in normal epithelial cells was localized in the cytoplasm as well as on the cell-cell boundary (Fig. 1d).

Relationship between SCCA2 and E-cadherin expression in primary cervical cancer lesions. We investigated the intensity and classification of SCCA2 and E-cadherin staining patterns in tissue sections from 70 primary cervical squamous cell carcinoma cases. Representative expression patterns of SCCA2 are shown in Fig. 2 (Strong pattern, Fig. 2a and b; W\&H pattern, Fig. 2c and d; Heterogeneous pattern, Fig. 2e and f) and of E-cadherin in Fig. 3 (Strong pattern, Fig. 3a and b; W\&H pattern, Fig. 3c and d; Heterogeneous pattern, Fig. 3e 

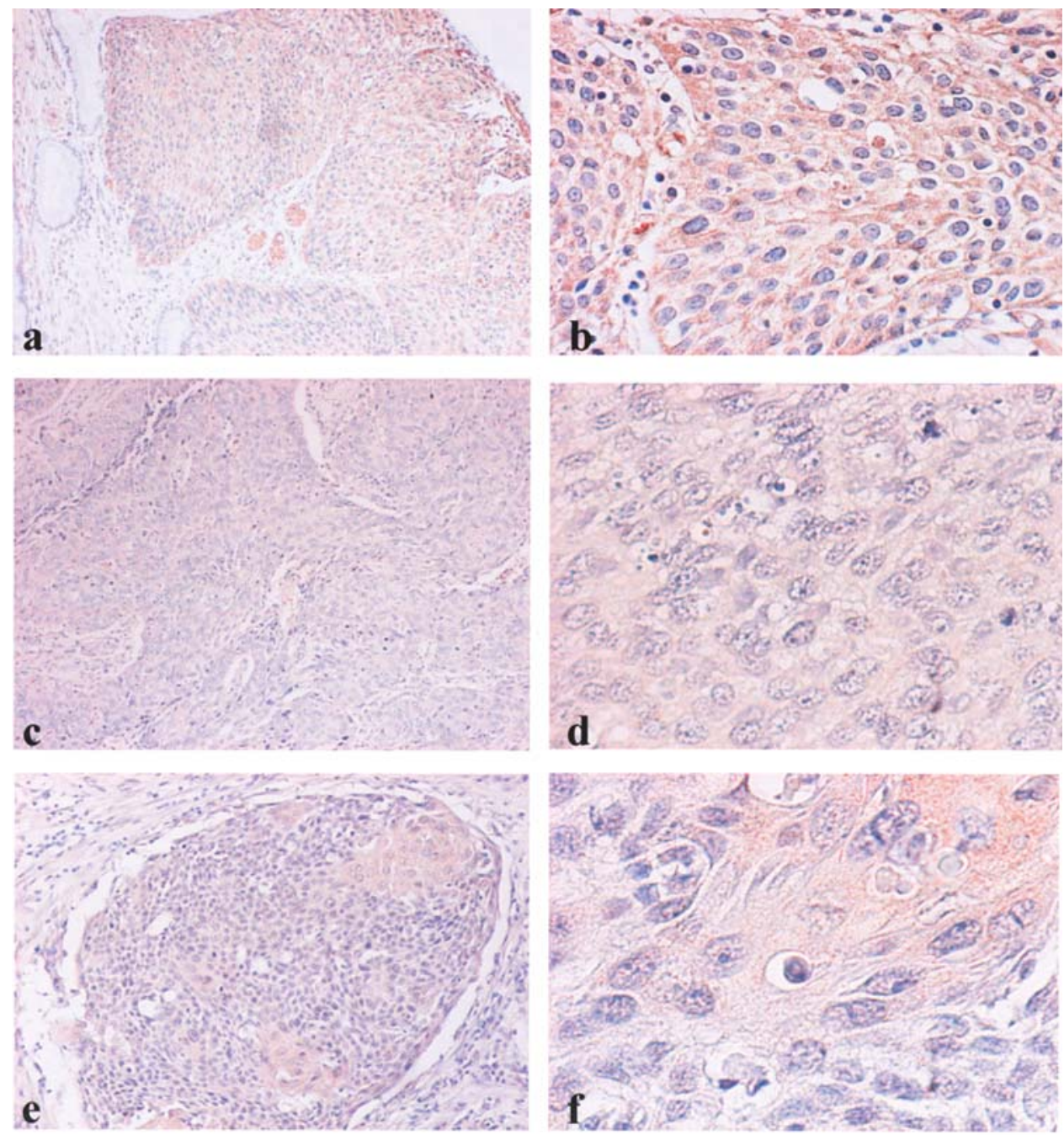

Figure 3. Expression pattern of E-cadherin in primary cervical squamous cell carcinoma by immunohistochemistry. (a, b) Strong pattern: the intensity of Ecadherin staining in cancer cells is similar to that in the normal squamous epithelial cells seen in Fig. 1. All cancer cells evaluated as (+). (c, d) W\&H pattern: E-cadherin staining in cancer cells is weaker than that in the normal epithelial cells. All cancer cells evaluated as $( \pm)$. (e, f) Heterogenous pattern: the intensity of E-cadherin staining differs from cell to cell. Some cancer cells evaluated as (+), ( \pm ), or (-). Original magnification (a, c and e), x100; (b, d and f), $\mathrm{x} 400$.

Table I. Relationship in expression patterns between SCCA2 and E-cadherin.

\begin{tabular}{lccr}
\hline & \multicolumn{2}{c}{ E-cadherin expression patterns } \\
\cline { 2 - 4 } & Strong & W\&H & Heterogeneous \\
\hline SCCA2 expression & patterns & & \\
Strong & 29 & 2 & 2 \\
W\&H & 2 & 9 & 2 \\
Heterogeneous & 4 & 4 & 19 \\
\hline
\end{tabular}

Spearman rank correlation test, $\mathrm{p}<0.001$.

and f). The results show a significant relationship in expression patterns between SCCA2 and E-cadherin (Table I, $\mathrm{p}<0.001)$.

Expression patterns of SCCA2 in primary cancer lesions and its relationship to lymph node metastasis. To examine the relationship between SCCA2 expression and lymph node metastasis, each immunohistochemical sample was analyzed as shown in Table II. Of the 70 cases, 33 exhibited Strong pattern, $13 \mathrm{~W} \& \mathrm{H}$ pattern and 24 Heterogeneous pattern. In 20 cases positive for lymph node metastasis, 14 exhibited Heterogeneous pattern and 4 Strong pattern whereas, in 50 cases without lymph node metastasis, 10 exhibited Heterogeneous pattern and 29 Strong pattern (Table II, $\mathrm{p}<0.01$ ). There was, however, no significant relationship between $\mathrm{W} \& \mathrm{H}$ pattern and lymph node metastasis (Table II).

Expression patterns of E-cadherin in primary cancer lesions and its relationship to lymph node metastasis. To examine the relationship between E-cadherin expression patterns and lymph node metastasis, we investigated the E-cadherin expression patterns of the same cases as above (Table III). Of the 70 cases, 35 exhibited Strong pattern, $15 \mathrm{~W} \& \mathrm{H}$ pattern and 20 Heterogeneous pattern. In the 20 cases positive for lymph node metastasis, 13 exhibited Heterogeneous pattern and 3 strong pattern whereas, in the 50 cases without lymph node metastasis, 7 exhibited Heterogeneous pattern and 32 strong pattern (Table III, $\mathrm{p}<0.001$ ). 
Table II. Expression patterns of SCCA2 in primary cancer lesions and lymph node metastasis.

\begin{tabular}{ccccc}
\hline & & \multicolumn{2}{c}{ SCCA2 expression patterns } \\
\cline { 3 - 4 } & No. & Strong & W\&H Heterogeneous \\
\hline $\begin{array}{c}\text { Metastasis } \\
(+)\end{array}$ & 20 & 4 & 2 & 14 \\
$(-)$ & 50 & 29 & 11 & 10 \\
& 70 & 33 & 13 & 24 \\
\hline
\end{tabular}

Fisher's exact test with Bonferroni correction: Strong vs. Heterogeneous, $\mathrm{p}<0.01$; Strong vs. W\&H, NS; Heterogeneous vs. W\&H, NS.

Table III. Expression patterns of E-cadherin in primary cancer lesions and lymph node metastasis.

E-cadherin expression patterns

\begin{tabular}{cccrr} 
& No. & Strong & W\&H & Heterogeneous \\
\hline Metastasis & & & & \\
$(+)$ & 20 & 3 & 4 & 13 \\
$(-)$ & 50 & 32 & 11 & 7 \\
& 70 & 35 & 15 & 20 \\
\hline
\end{tabular}

Fisher's exact test with Bonferroni correction: Strong vs. Heterogeneous, $\mathrm{p}<0.001$; Strong vs. W\&H, NS; Heterogeneous vs. W\&H, NS.

\section{Discussion}

The present study shows that there is a significant relationship between SCCA2 and E-cadherin expression patterns in squamous cell carcinoma tissues, and that the Heterogeneous pattern they exhibit in primary lesions is strongly related to a high incidence of lymph node metastasis. The present data suggest that cancer cells lacking SCCA and E-cadherin expression have a potential for lymph node metastasis because the presence of the Heterogeneous pattern, including cancer cells exhibiting the loss of SCCA and E-cadherin, is related to lymph node metastasis, but Strong and W\&H patterns with SCCA2 and E-cadherin expression are not. We recently found that the suppression of SCCA2 expression in squamous cell carcinoma cells in vitro promotes cancer cell invasion and migration accompanied by decreased E-cadherin expression (18), suggesting that SCCA may be involved in metastasis through the alteration of E-cadherin expression. We therefore can hypothesize that cancer cells which have lost SCCA2 expression metastasize to lymph nodes through the decrease in E-cadherin expression.

E-cadherin is one of the most important adhesion molecules in the epithelium, mediating cell-to-cell contact. Altered expression of E-cadherin is involved in cancer behavior, particularly in the process of cancer invasion and metastasis.
A number of studies have reported that the loss of E-cadherin plays an important role in the progression of cancer cells and is associated with their metastasis $(13-17,19)$. In fact, we have shown that the blockage of E-cadherin functions by anti-E-cadherin antibody treatment promotes cell invasion and migration in squamous cell carcinoma cell lines (18). Some reports have also referred to the relationship between E-cadherin expression and lymph node metastasis in esophageal $(14,15)$, gastric $(20,21)$, endometrial $(22)$, breast $(23,24)$, head and neck $(13,16)$ and skin cancer $(17)$. This is, however, the first report on the relationship between E-cadherin and lymph node metastasis in uterine cervical squamous cell carcinoma and, moreover, on the relationship between SCCA expression and lymph node metastasis.

It has been reported that SCCA is immunohistochemically strongly positive in well-differentiated squamous cell carcinoma cells (25). The same is true of E-cadherin, which is, however, negative in poorly-differentiated cells $(13,19)$. The present study has shown that, when the SCCA2 expression pattern in primary cancer lesions is heterogeneous, sensitivity and specificity for predicting lymph node metastasis are 70 and $80 \%$, respectively. In case of E-cadherin expression of Heterogenous pattern, sensitivity and specificity for predicting lymph node metastasis are 65 and 86\%, respectively. Analyses of SCCA2 and E-cadherin expression patterns in primary cervical cancer lesions may be useful new markers for the prediction of lymph node metastasis.

In summary, the present study has resulted in a new finding on the relationship between SCCA2 and E-cadherin expression in vivo, namely that the presence of Heterogeneous pattern in the two is closely related to lymph node metastasis. Cancer cells with loss of SCCA2 and E-cadherin expression may actually cause lymph node metastasis. This possibility is supported by our previous findings, that the suppression of SCCA2 promotes cancer cell invasion and migration through a decrease in E-cadherin expression (18). This study gives insight into the mechanism between cell adhesion and the malignant behavior of cancer cells.

\section{Acknowledgements}

We thank Dr Gary A. Silverman for providing the specific antibodies for SCCA2. This work was supported by a Grantin-Aid for Scientific Research on Priority Areas (C-2) from the Ministry of Education, Science, Sports and Culture (12218224, 16591664) and by a Grant-in-Aid for Second Term Comprehensive 10-year Strategy for Cancer Control from the Ministry of Health and Welfare (H12-Gan-025), Japan.

\section{References}

1. Kato $\mathrm{H}$ and Torigoe $\mathrm{T}$ : Radioimmunoassay for tumor antigen of human cervical squamous cell carcinoma. Cancer 40: 1621-1628, 1977.

2. De Bruijn HWA, Duk JM, van der Zee AG, Pras E, Willemse PHB, Boonstra H, Hollema H, Mourits MJE, De Vries EGE and Aalders JG: The clinical value of squamous cell carcinoma antigen in cancer of the uterine cervix. Tumor Biol 19: 505-516, 1998 .

3. Schneider SS, Schick C, Fish KE, Miller E, Pena JC, Treter SD, Hui SM and Shilverman GA: A serine proteinase inhibitor locus at 18q21.3 contains a tandem duplication of human squamous cell carcinoma antigen gene. Proc Natl Acad Sci USA 92: 3147-3151, 1995 
4. Suminami Y, Kishi F, Sekiguchi K and Kato H: Squamous cell carcinoma antigen is a new member of the serine protease inhibitors. Biochem Biophys Res Comm 181: 51-58, 1991.

5. Nawata S, Tsunaga N, Numa F, Tanaka T, Nakamura K and Kato $\mathrm{H}$ : Serine protease inhibitor activity of recombinant squamous cell carcinoma antigen towards chymotypsin, as demonstrated by sodium dodecyl sulfate-polyacrylamide gel electrophoresis. Electrophoresis 16: 1027-1030, 1995.

6. Nawata S, Nakamura K, Tanaka T, Numa F, Suminami Y, Tsunaga N, Kakegawa H, Katunuma N and Kato H: Electrophoretic analysis of the 'cross-class' interaction between novel inhibitory serpin, squamous cell carcinoma antigen-1 and cystein proteinases. Electrophoresis 18: 784-789, 1997.

7. Schick C, Kamachi Y, Bartuski AJ, Cataltepe S, Schechter NM, Pemberton PA and Silverman GA: A squamous cell carcinoma antigen 2 is a novel serpin that inhibits the chymotrypsin-like proteinase cathepsin $\mathrm{G}$ and mast cell chymase. J Biol Chem 272: 1849-1855, 1997.

8. Schick C, Pemberton PA, Shi GP, Kamachi Y, Catalpete S, Bartuski AJ, Gornstein ER, Brömme D, Chapman HA and Silverman GA: Cross-class inhibition of the cysteine proteinases cathepsin $\mathrm{K}, \mathrm{L}$, and $\mathrm{S}$ by serpin squamous cell carcinoma antigen 1: a kinetic analysis. Biochemistry 37: 5258-5266, 1998.

9. Murakami A, Suminami Y, Hirakawa H, Nawata S, Numa F and Kato H: Squamous cell carcinoma antigen suppresses radiationinduced cell death. Br J Cancer 84: 851-858, 2001.

10. Murakami A, Suminami Y, Sakaguchi Y, Nawata S, Numa F, Kishi $\mathrm{F}$ and Kato H: Specific detection and quantitation of SCC antigen 1 and SCC antigen $2 \mathrm{mRNAs}$ by fluorescence-based asymmetric semi-nested reverse transcription PCR. Tumor Biol 21: 224-234, 2000.

11. Gariboldi M, Peissel B, Fabbri A, Saran A, Zaffaroni D, Falvell FS, Spinola M, Tamura J, Pazzaglia S, Mancuso MT, Maurichi A, Bartoli C, Cataltepe S, Silverman GA, Pilotti S, Hayashizaki Y, Okazaki Y and Dragani TA: SCCA2-like serpins mediate genetic predisposition to skin tumors. Cancer Res 63: 1871-1875, 2003

12. Liotta LA: Tumor invasion and metastasis: Role of the extracellular matrix. Cancer Res 46: 1-7, 1986.

13. Schipper JH, Frixen UH, Behrens J, Unger A, Jahnke K and Birchmeier W: E-cadherin expression in squamous cell carcinomas of head and neck: inverse correlation with tumour dedifferentiation and lymph node metastasis. Cancer Res 51: 6328-6337, 1991.

14. Shiozaki H, Tahara H, Oka H, Miyata M, Kobayashi K, Tamura S, Iihara K, Doki Y, Hirano S, Takeichi M and Mori T: Expression of immunoreactive E-cadherin adhesion molecules in human cancers. Am J Pathol 139: 17-23, 1991.
15. Kadowaki T, Shiozaki H, Inoue M, Tamura S, Oka H, Doki Y, Iihara K, Matsui S, Iwazawa T, Nagafuchi A, Tsukita S and Mori T: E-cadherin and $\alpha$-catenin expression in human esophageal cancer. Cancer Res 54: 291-296, 1994.

16. Bowie GL, Caslin AW, Roland NJ, Field JKMA, Jones AS and Kinsella AR: Expression of the cell-cell adhesion molecule E-cadherin in squamous cell carcinoma of the head and neck. Clin Otolaryngol 18: 196-201, 1993.

17. Koseki S, Aoki T, Ansai S, Hozumi Y, Mitsuhashi Y and Kondo S: An immunohistchemical study of E-cadherin expression in human squamous cell carcinoma of the skin. J Dermatol 26: 416-422, 1999.

18. Murakami A, Nakagawa T, Kaneko M, Nawata S, Takeda O, Kato H and Sugino N: Suppression of SCC antigen promotes cancer cell invasion and migration through the decrease in Ecadherin expression. Int J Oncol 29: 1231-1235, 2006.

19. Wu H, Lotan R, Mente D, Lippman SM and Xu XC: Expression of E-cadherin is associated with squamous differentiation in squamous cell carcinomas. Anticancer Res 20: 1385-1390, 2000.

20. Mayer B, Johnson JP, Leitl F, Jauch KW, Heiss MM, Schildberg FW, Birchmeier W and Funke I: E-cadherin expression in primary and metastatic gastric caner: Downregulation correlates with cellular dedifferentiation and glandular disintegration. Cancer Res 53: 1690-1695, 1993.

21. Cai Jinhui, Ikeguchi M, Ysujitani S, Maeta M, Liu J and Kaibara N: Significant correlation between micrometastasis in the lymph nodes and reduced expression of E-cadherin in early gastric cancer. Gastric Cancer 4: 66-74, 2001.

22. Sakuragi N, Nishiya M, Ideda K, Ohkouch T, Furth EE, Hareyama H, Satoh C and Fujimoto S: Decreased E-cadherin expression in endometrial carcinoma is associated with tumor dedifferentiation and deep myometrial invasion. Gynecol Oncol 53: 183-189, 1994.

23. Oka H, Shiozaki H, Kobayashi K, Inoue M, Tahara H, Kobayashi T, Takatsuka Y, Matsuyoshi N, Hirano S, Takeuchi M and Mori T: Expression of E-cadherin cell adhesion molecules in human breast cancer tissues and its relationship to metastasis. Cancer Res 53: 1696-1701, 1993.

24. Jones JL, Raoyall JE and Walker RA: E-cadherin relates to EGFR expression and lymph node metastasis in primary breast carcinoma. Br J Cancer 74: 1237-1241, 1996.

25. Hoshina M, Kimura A, Shibata K, Maruo T and Mochizuki M Immunocytological distribution of the tumor antigen TA-4: expression during carcinogenesis and maturation of squamous epithelium of human uterine cervix. Asia-Oceania J Obstet Gynecol 12: 119-126, 1986. 